\title{
Erratum to: Hypercholesterolemia downregulates autophagy in the rat heart
}

\author{
Zoltán Giricz ${ }^{1,5^{*}}$, Gábor Koncsos ${ }^{1}$, Tomáš Rajtík ${ }^{3}$, Zoltán V. Varga ${ }^{1,2}$, Tamás Baranyaii ${ }^{1}$, Csaba Csonka ${ }^{2}$, Adrián Szobi ${ }^{3}$, \\ Adriana Adameová ${ }^{3}$, Roberta A. Gottlieb ${ }^{4}$ and Péter Ferdinandy ${ }^{1,5}$
}

\section{Erratum}

Following publication of the original article [1], it came to the attention of authors that the definition of the term "isolated hypercholesterolemia" used frequently in the article is missing. In this paper, authors define isolated hypercholesterolemia as a hypercholesterolemia without an accompanying hypertriglyceridemia. Therefore, we would like to amend the Background paragraph of the Abstract with the following sentence: "However, it is unknown whether isolated hypercholesterolemia (i.e., hypercholesterolemia without hypertriglyceridemia) disturbs autophagy or the mammalian target of rapamycin (mTOR) pathways."

In addition, the second sentence of Background section was also incorrectly phrased; which we would like to correct as follows: "The role of atherosclerosis is well studied in these pathologies; however, myocardial effects of hypercholesterolemia is less well understood."

\begin{abstract}
Author details
'Department of Pharmacology and Pharmacotherapy, Faculty of Medicine, Semmelweis University, Nagyvárad tér 4, Budapest H-1089, Hungary.

${ }^{2}$ Department of Biochemistry, Faculty of Medicine, University of Szeged, Dómtér 9, Szeged H-6720, Hungary. ${ }^{3}$ Department of Pharmacology and Toxicology, Faculty of Pharmacy, Comenius University, Odbojárov 10, 83232 Bratislava, Slovakia. ${ }^{4}$ Heart Institute, Cedars-Sinai Medical Center, 127 S. San Vicente Blvd, Los Angeles, CA 90048, USA. ${ }^{5}$ Pharmahungary Group, Szeged, Hungary.
\end{abstract}

Received: 22 June 2017 Accepted: 22 June 2017

Published online: 05 July 2017

\section{Reference}

1. Giricz Z, Koncsos G, Rajtík T, Varga ZV, Baranyai T, Csonka C, et al. Gottlieb and Péter Ferdinandy. Lipids in Health and Disease. 2017;16:60. doi:10.1186/ s12944-017-0455-0.

\footnotetext{
* Correspondence: giricz.zoltan@med.semmelweis-univ.hu

'Department of Pharmacology and Pharmacotherapy, Faculty of Medicine,

Semmelweis University, Nagyvárad tér 4, Budapest H-1089, Hungary

${ }^{5}$ Pharmahungary Group, Szeged, Hungary
} 